\title{
Epicardial Fibrosis explains Increased Transmural Conduction in a Computer Model of Atrial Fibrillation
}

\author{
Ali Gharaviri ${ }^{1}$, Mark Potse ${ }^{1,2}$, Sander Verheule ${ }^{3}$, Rolf Krause ${ }^{1}$, Angelo Auricchio ${ }^{1,4}$, Ulrich Schotten ${ }^{3}$ \\ ${ }^{1}$ Center for Computational Medicine in Cardiology, Università della Svizzera italiana, Lugano, \\ Switzerland. ${ }^{2}$ Inria Bordeaux, France. ${ }^{3}$ Department of Physiology, Maastricht University, \\ Maastricht, The Netherlands. ${ }^{4}$ Cardiocentro Ticino, Lugano, Switzerland.
}

\begin{abstract}
Recent work has shown that the transition from persistent to permanent $A F$ in goats coincides with an increase in fibrosis in the outer millimeter of the atrial wall. Macroscopically this leads to reduced electrical conductivity orthogonal to the dominant fiber orientation.

We constructed a detailed geometry of the human atria including epicardial layer and all major endocardial bundle structures. The model also includes realistic one to three layers of fiber orientations, corresponding to their location in the atrium.

The numbers of waves, phase singularities, and breakthroughs (BTs) were quantified at different degrees of fibrotic tissue. Increase in the "fibrotic" volume from zero (Control) to moderate (50\% Fibrotic), and severe (70\% Fibrotic) increased both the number of waves and the number of phase singularities. Along with the increase in fibrosis, the endo-epicardial electrical activity dyssynchrony increased.
\end{abstract}

\section{Introduction}

The perpetuation and progression of atrial fibrillation (AF) is mediated by electrical and structural remodeling the atria [1]. Electrical remodeling is a relatively fast process (1-2 days) that includes action potential shortening and reduction of effective refractory period. Structural remodeling develops much more slowly (months-years) and is considered as the main mechanism leading to AF stability. Several studies have demonstrated that structural remodeling is associated with a decrease in the size of fibrillatory waves and an increased incidence of transmural conduction ("breakthroughs") in both patients and goat models [2-4]. Verheule et al. demonstrated that transition from persistent to permanent AF in goats is histologically characterized by an increase in endomysial fibrosis, particularly in the outer millimeter of the atrial wall [2]. In this way, endo-epicardial transmural connectivity and electrical synchronization between endo- and epicardium would be hampered. Using simultaneous endo-epicardial mapping, Eckstein et al. showed a significant increase in endo-epicardial electrical dissociation (EED) as well as breakthroughs (transmurally propagating fibrillation waves) in goats in later stages of AF [5,6].

While these experimental studies show a clear association between structural remodeling, EED, and breakthrough incidence, they cannot establish a causal relationship or elucidate which aspects of the structural remodeling are responsible for the observed changes. The purpose of this study was to assess the effects of loss of side-to-side connectivity in the epicardial layer on EED and breakthrough incidence, using a numerical model in which all other factors were unchanged.

\section{Method}

\section{1. $\quad 3 D$ model structure}

An anatomical 3D model of human atria including fiber orientation was built manually using Blender (The Blender Foundation, Amsterdam, The Netherlands) as a 3D editing tool. The global shape was extracted from magnetic resonance imaging data of a subject with normal atrial anatomy. Endocardial and epicardial surfaces were created and combined into a 'myocardial envelope': a closed surface containing the atrial myocardium. 20 Pectinate muscles (PM) in the right atrial (RA) free wall, Bachmann's Bundle (BB), inter atrial bundles, and the crista terminalis (CT) were added based on published anatomical descriptions [7-9]. The model contained four pulmonary veins, caval veins, left and right atrial appendages (LAA and RAA), as well as the coronary sinus, as illustrated in figure $1 \mathrm{~A}, \mathrm{~B}$ and $\mathrm{C}$.

Dedicated software was used to create a computational mesh at $0.2-\mathrm{mm}$ resolution with different fiber orientations from the manually-edited objects. Within the myocardial envelope a 1-mm layer of myocardium was placed to represent the thin right-atrial wall. A 3-mm layer was used for the thicker left atrium. The bundles consisted of myocardium with different conduction properties. 


\subsection{Fiber orientation and anisotropy}

One of the most important properties of this model is the incorporation of realistic fiber orientation based on histological observations (see figure 1C and D) [7]. Ho et al have described the left-atrial wall as 2 layers with different fiber orientations [7]. Less information is available for the right atrium.

In our model, elements within the bundles received a fiber orientation parallel to the local bundle orientation.

\subsection{Electrophysiological model}

Simulations were performed with the propag- 5 software and run on 2560 cores of a Cray XE6 supercomputer [10, 11]. Electrical activity was simulated with a mono-domain reaction-diffusion equation. Ionic currents and calcium handling for each model node was described by Courtemanche-Ramirez-Nattel model [12].

To reproduce electrical remodeling as observed in atrial fibrillation, conductivities for $\mathrm{I}_{\mathrm{to}}, \mathrm{I}_{\mathrm{caL}}$, and $\mathrm{I}_{\mathrm{k} 1}$ were set at $15 \%, 15 \%$, and $200 \%$ of their normal values respectively [1].

\subsection{Simulation protocol}

A spiral was initiated via a single pacing site in combination with an associated temporary block line.

\subsection{Implementation of fibrosis}

Fibrosis segments were simulated by randomly removing transverse conductivity between elements. Three different degrees of fibrosis (slight (50\%), moderate $(60 \%)$, and severe (70\%)) were selected and simulated to be compared with no fibrosis (control).
A)

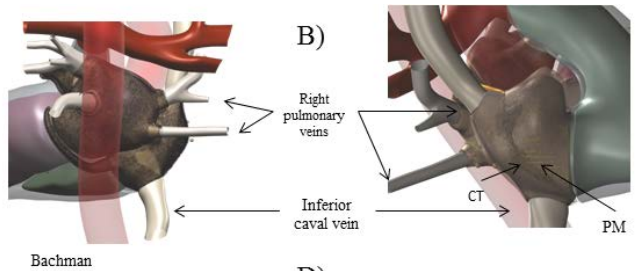

C)
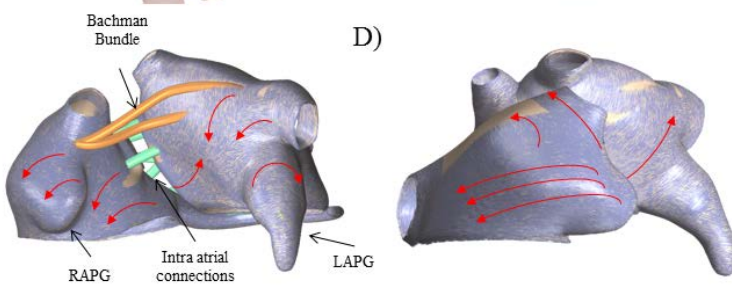

Figure 1. Anatomical model of the atria used for simulations. A) Posterior view. B) Saggital view. C) Fiber orientations in anterior view. D) RA free wall fiber orientations.

\section{Analysis}

\subsection{Detection of phase singularities}

In two dimensions, the tip of a reentrant wave forms a phase singularity (PS); a point where all phases of the action potential simultaneously occur. The threedimensional equivalent of a PS is a vortex filament. Model elements that were part of vortex filaments were detected using the method [13]. A filament was defined by the line of intersection of the two surfaces $V(r, t)=V i s o$, where $V i s o$ is an arbitrary selected potential value which used to distinguish polarized and depolarized regions of the tissue (in this study $-49 \mathrm{mV}$ ), and $\partial_{t} V(r, t)=0$. Since in our simulations the time is discrete we could approximate the $\partial_{t} V(r, t)=0$ by $\partial_{t} V(r, t) \approx V(r,(n+1) \Delta t)-$

$V(r, n \Delta t)$. Therefore, the filament position could be identified using formula (1).

$$
V(r, n \Delta t)=V(r,(n+1) \Delta t)=V_{\text {iso }}
$$

In order to describe this method more practically we represent the filament line by the set of intersection points of this line with the lattice planes defined $x=\Delta i, \mathrm{y}=$ $\Delta j, z=k \Delta z$, which they are all the $y-z, x-z$, and $x-y$ planes that contain lattice points, respectively. Since each plane is made up of squares with one lattice point at each of the four corners, the algorithm proceeds by finding which of the $3 \mathrm{NxNyNz}$ squares contained in these planes are intersected by the line defined by Eq. (1) and what are the coordinates of these intersection [13]. Finally, all of these points are sorted in order of increasing arclength $S$ along a given filament, the arclength distance between two nearby points $\left(p\left(p_{1}, p_{2}, p_{3}\right)\right.$ and $\left.q\left(q_{1}, q_{2}, q_{3}\right)\right)$ being simply defined by the Euclidean distance $(d(p, q)=$ $\left.\sqrt{\left(p_{1}-q_{1}\right)^{2}+\left(p_{2}-q_{2}\right)^{2}+\left(p_{3}-q_{3}\right)^{2}}\right)$.

\subsection{Detection of fibrillation waves}

A wave was defined as a contiguous area in which all nodes have trans-membrane voltages above the excitation threshold of $-60 \mathrm{mV}$. The number of waves was calculated each millisecond of simulated time.

\subsection{Dyssynchrony}

Dyssynchrony was defined as the percentage of nodes that were excited (membrane potential above $-60 \mathrm{mV}$ ), while the opposing segment of the atrial wall was not excited (membrane potential below $-60 \mathrm{mV}$ ).

In order to calculate dyssynchrony, model nodes were categorized either as epicardial or endocardial. Pairs of endocardial and epicardial nodes were created by finding the nearest epicardial node for each endocardial node. 
Dyssynchrony was defined as the number of pairs with different state (one node activated and the other not activated) divided by the total number of pairs. Dyssynchrony was computed for each millisecond of simulated time.

\subsection{Detection of breakthroughs}

A breakthrough (BT) is a wave that appears in epicardial layer and cannot be related to the propagation of other waves in that layer. To detect BTs, wave sizes at each time step were calculated. If a wave smaller than a threshold (9 nodes in this study) appears within the epicardial layer and this wave has an overlap with a wave in endocardial layer, this was considered as a BT candidate. Each BT candidate was monitored and if its size increased in two folds, it was confirmed as a BT.

\section{Results}

\subsection{Effect of fibrosis}

\subsubsection{Fibrillation patterns}

In figure 2, an example of fibrillation patterns in both control and severe fibrosis are depicted. In the presence of fibrosis, fibrillation patterns in RA, LA anterior wall, and pulmonary veins area were more complex compared to control. Longitudinal dissociation, electric uncoupling of side-to-side connections between parallel-oriented atrial muscle bundles, within the epicardial plane increased and waves became narrower.
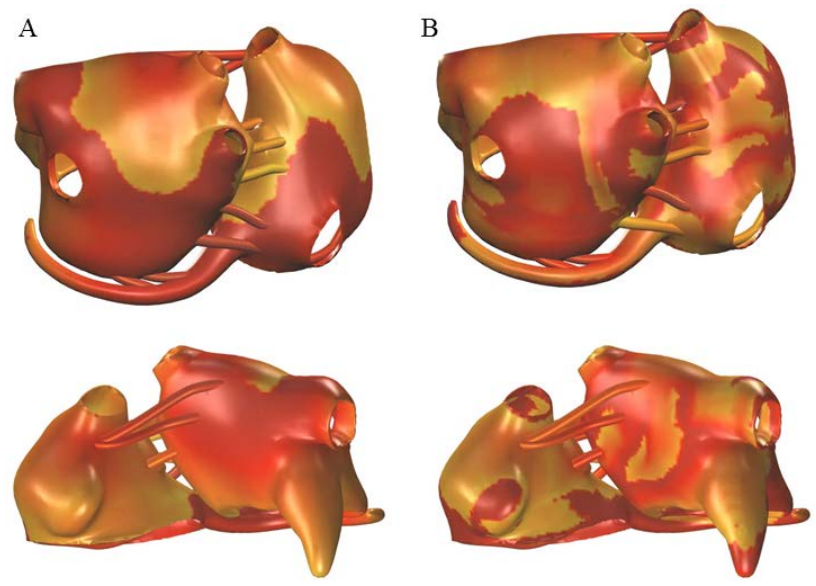

Figure 2. A) Potential map in LA and RA (control). B) Potential map in LA and RA (severe fibrosis).

\subsubsection{Dissociation and BT}

Figure 3 illustrated examples of conduction patterns in control (3A and C) and severe fibrosis (3B and D). As illustrated in this figure in both cases (control and severe fibrosis) BTs occurred but incidence of BTs in control group was more repetitive and limited to only few locations. An increase in the degree of fibrosis enhanced endo-epicardial electrical dissociation which increased the chance for BT occurrence and therefore more BTs were observed at different locations. For example, in none of the control group simulations a BTs was observed in RA free wall near the PMs or in anterior side of LA but multiple breakthroughs occurred at those sites in the presence of fibrosis (figure $3 \mathrm{~B}$ and $\mathrm{D}$ ).

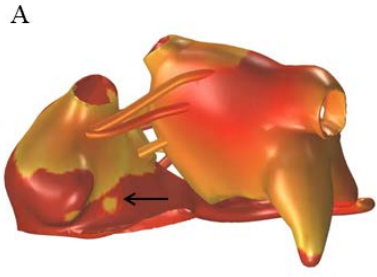

C

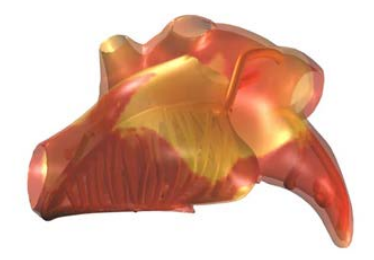

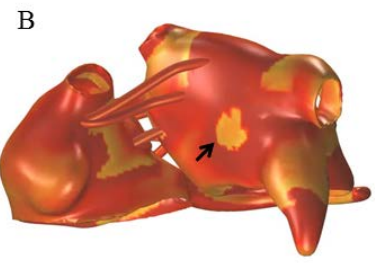

D

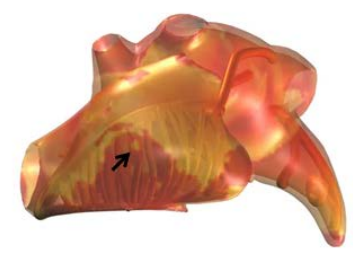

Figure 3. A) Incidence of BT in (black arrow) in control simulation. B) Incidence of BT (black arrow) in severe fibrosis simulation. C) Electrical activity association between PMs and right atrial epicardial layer. D) Increase in electrical activity dissociation in PMs and right atrial epicardial layer and incidence of BT.

\subsubsection{Quantitative analysis of fibrillation patterns}

The numbers of waves, phase singularities, and breakthroughs (BTs) were quantified at different degrees of fibrotic tissue. Increase in the "fibrotic" volume from zero (Control) to slight (50\%), moderate (60\% Fibrotic), and severe (70\% Fibrotic) increased both the number of waves and the number of phase singularities. Along with the increase in fibrosis, the endo-epicardial electrical activity dyssynchrony and the incidence of BTs increased (see figure 4). 


\section{Discussion}

In this study we present a highly detailed 3D anatomical model of the human atria. This model was used to test the hypothesis that fibrosis in the epicardial layer would be sufficient to produce increased endo-epicardial dissociation of electrical activity and as a consequence an increase in transmural conduction. Our simulations demonstrate that epicardial fibrosis increased the number of waves and PSs, endo-epicardial dissociation of electrical activity and the BT rate.
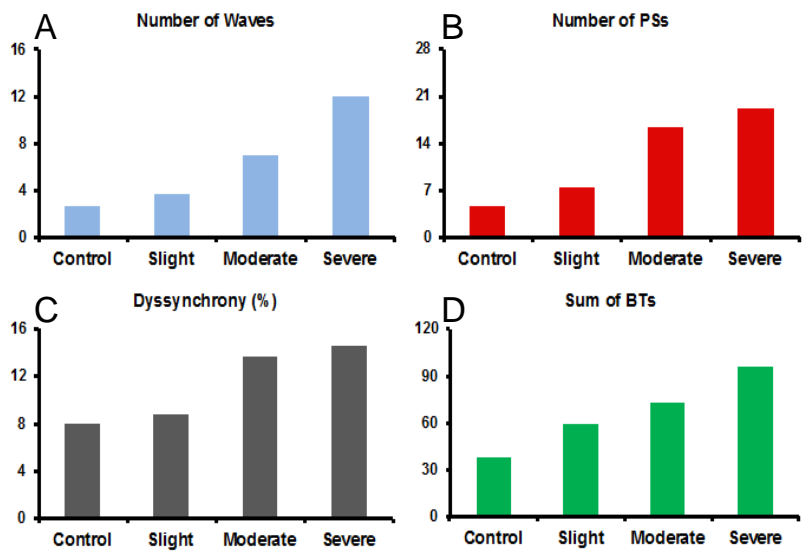

Figure 4. A) Number of waves, B) phase singularities, C) percentages of endo-epicardial electrical activity dissociation, and D) summation of all occurred BTs during simulation period in different degrees of fibrosis.

\section{Study limitations}

There were some limitations regarding this study. (1) Human experimental and histological data were sparse and limited e.g. the histological data regarding fiber orientation in the RA of the human heart is very limited. For this reason, the fiber orientation in the RA thin wall was implemented using derived histological data from goats. (2) Several studies have proposed AP heterogeneity as a main mechanism for AF initiation and perpetuation, but in this model only one myocyte model with the same characteristics was used in different region of atrium. Our model offers an excellent opportunity to investigate the presence of this property in the future. (3) Random focal discharges, which have been investigated in several $\mathrm{AF}$ computer models and can be one of the important AF mechanisms, are also not implemented in this model [14].

\section{References}

[1] Schotten U, Verheule S. Pathophysiological mechanisms of atrial fibrillation: a translational appraisal. Physiological reviews 201;1:265-325.

[2] Verheule S, Tuyls E. Fibrillatory conduction in the atrial free walls of goats in persistent and permanent atrial fibrillation. Circulation Arrhythmia and electrophysiology 2010;3:590599.

[3] de Groot NM, Houben RP. Electropathological substrate of longstanding persistent atrial fibrillation in patients with structural heart disease: epicardial breakthrough. Circulation 2010;122:1674-1682.

[4] Allessie MA, de Groot NM. Electropathological substrate of long-standing persistent atrial fibrillation in patients with structural heart disease: longitudinal dissociation. Circulation Arrhythmia and electrophysiology 2010;3:606615.

[5] Eckstein J, Maesen B. Time course and mechanisms of endoepicardial electrical dissociation during atrial fibrillation in the goat. Cardiovascular research 2011;89:816-824.

[6] Eckstein J, Zeemering S. Transmural conduction is the predominant mechanism of breakthrough during atrial fibrillation: evidence from simultaneous endo-epicardial high-density activation mapping. Circulation Arrhythmia and electrophysiology 2013;6:334-341.

[7] Ho SY, Anderson RH. Atrial structure and fibres: morphologic bases of atrial conduction. Cardiovasc Res 2002;54:325-336.

[8] Ho SY, Sanchez-Quintana D. The importance of atrial structure and fibers. Clinical Anatomy. 2009;22:52-63.

[9] Anderson R, Becker AE. Cardiac Anatomy: An Integrated Text and Colour Atlas. Gower Medical Publishing, London 1980.

[10] Potse M, Dube B, Richer J, Vinet A, Gulrajani RM. A comparison of monodomain and bidomain reactiondiffusion models for action potential propagation in the human heart. IEEE transactions on bio-medical engineering Dec 2006;53:2425-2435.

[11] Krause D, Potse M, Dickopf T, Krause R, Auricchio A, Prinzen F. Hybrid Parallelization of a Large-Scale Heart Model. In: Keller R, Kramer D, Weiss J-P, eds. Facing the Multicore - Challenge II. Vol 7174: Springer Berlin Heidelberg; 2012:120-132.

[12] Courtemanche $\mathrm{M}$, Ramirez RJ. Ionic mechanisms underlying human atrial action potential properties: insights from a mathematical model. The American journal of physiology1998;275:H301-321.

[13] Fenton F, Karma A. Vortex dynamics in three-dimensional continuous myocardium with fiber rotation: Filament instability and fibrillation. Chaos 1998;8:20-47.

[14] Gong Y, Xie F, Stein KM. Mechanism underlying initiation of paroxysmal atrial flutter/atrial fibrillation by ectopic foci: a simulation study. Circulation 2007;115:2094-2102.

Address for correspondence.

Ali Gharaviri

Institute of Computational Science, Faculty of Informatics

Via Giuseppe Buffi 13,6900 Lugano, Switzerland

ali.gharaviri@usi.ch 